\title{
Safe distance, safe patients! Therapeutic management of oncological patients affected by cutaneous and mucosal adverse events during the COVID-19 pandemic: an Italian experience
}

\author{
Eleonora Cinelli ${ }^{1}$ (D) $\cdot$ Gabriella Fabbrocini $^{1} \cdot$ Davide Fattore $^{1} \cdot$ Claudio Marasca $^{1} \cdot$ Giovanni Damiani $^{2,3}$. $^{2}$ \\ Maria Carmela Annunziata ${ }^{1}$
}

Received: 14 April 2020 / Accepted: 2 June 2020 / Published online: 8 June 2020

(C) Springer-Verlag GmbH Germany, part of Springer Nature 2020

\begin{abstract}
Stringent measures have been taken to contain COVID-19 spread, limiting access only for urgent visits, surgery procedures, or hospitalizations and using teledermatology services for non-urgent cases. Management of oncological patients affected by chemo-, immune-, and radiotherapy-related cutaneous and mucosal adverse events is a challenge. Firstly because of the differential diagnosis of cutaneous rash (e.g., drug-related rash or paraviral exanthema). Secondly, oncological patients can suffer from xerosis, pruritus, and mucositis that contribute to cutaneous and mucosal barrier lesions, thus becoming vulnerable site for viral or bacterial colonization. These lesions can also be aggravated by the use of protective mask and gloves. Here, we report also our results of a teledermatological survey on 87 oncological patients, where the health status of oncological patients referred to our dedicated clinic was assessed during the COVID-19 pandemic. Therefore, it is fundamental that oncological patients are followed up by their dermatologists even if the clinics are closed. Teledermatology represents a crucial means of communication. Patients can contact the dermatological staff by emails and telephone, $24 \mathrm{~h}$ a day, 7 days a week, for video calls and dermatological consultations.
\end{abstract}

Keywords Teledermatology $\cdot$ COVID-19 $\cdot$ Adverse events $\cdot$ Skin

Dear Editor,

From February 2020, Italy has been the center of one of the most severe cluster of coronavirus disease 19 (COVID-19) in the world [1], with a total of 150,189 cases on April 13, 2020 [2].

To date, stringent measures have been taken to contain infection spread in many countries around the world, including quarantine, and limiting as much as possible gatherings of people.

Eleonora Cinelli elecinelli@gmail.com

1 Section of Dermatology - Department of Clinical Medicine and Surgery, University of Naples Federico II, Via Pansini 5, 80131 Naples, Italy

2 Clinical Dermatology, IRCCS Istituto Ortopedico Galeazzi, Milan, Italy

3 Department of Biomedical, Surgical and Dental Sciences, University of Milan, Milan, Italy
These rules are particularly restrictive for hospitals in order to protect healthcare workers' (HCWs) and patients' health. For example, our Dermatological Department limited access only for urgent visits, surgery procedures, or hospitalizations [3], using teledermatology services for non-urgent cases.

Since 2010, our Department includes a specific outpatient clinic dedicated to chemo-, immune-, and radiotherapy-related cutaneous and mucosal adverse events (AEs). In oncological patients, managing approaches during the COVID-19 outbreak is a complicated challenge and actual available data on these people are conflicting. As reported by a nationwide analysis in China, oncological patients might have a higher risk of severe COVID-19 than subjects without cancer, thus the authors suggest a careful attention in the former patients' group [4]. However, a more recent paper by Xia and colleagues affirms that available evidences are not sufficient to conclude for an association between cancer and COVID-19 [5].

In our experience, oncological patients' management is still a challenge that both patients and dermatologists must face and fight. 
Table 1 Teledermatology survey: study population data

Patients, $n=105$

Patients contacted by telephone or video call
- Patients with worsening condition who required a shift or an increased dose of therapy*
- Patients with stable condition
- Patients with improving condition
- Patients in good health, only on preventive follow-up
Patients contacted by email
- Patients with worsening condition who required a shift or an increased dose of therapy
- Patients with stable condition
- Patients with improving condition
- Patients in good health, only on preventive follow-up
Patients who did not answer to both means of communications

35

8

12

8

7

52

6

21

12

13

18

*Topical and/or systemic therapy prescribed for chemotherapy- or immunotherapy-related adverse events in the previous visit

First of all, the development of a skin toxicity during anticancer therapies is not a simple one-way route. For example, a rash could be drug-related, as it typically occurs during chemotherapy or immunotherapy or, on the other hand, it could be a sort of tell-tale for a viral infection. A recent article by Recalcati showed that on eighteen COVID-19-positive patients, eight developed a cutaneous rash at the onset, with either erythematous, urticarial, or chicken-pox-like clinical feature [6]. The same clinical situations (especially the first two) can be noticed during drug reactions, as chemotherapy- or immunotherapyrelated AEs [7].

Second, cutaneous rash can commonly lead to xerosis and pruritus, and inflammation can occur also in the mucosae (mucositis). Both events can contribute to cutaneous and mucosal barrier lesions. Particularly, if the nasal mucosa is damaged, it may represent a vulnerable site for viral or bacterial colonization [8]. Moreover, we experienced that oncological patients, who frequently have a fragile health state, tend to use personal protective equipment (PPE) more frequently and for longer periods everyday during the COVID-19 outbreak to protect themselves from potential exposure to microbiological agents, and even more frequently and for longer periods during the COVID-19 outbreak. Although their timing of PPE use is not superimposable to those of HCWs, we cannot exclude that they are prone to develop the same skin and mucosa barrier damages due, for example, to frequent hand washing and PPE donning [8]. Nowadays in some Italian regions, it is even mandatory to use means of protection for nose, mouth, and hands during any possible shopping or outside activity. The use of the mask, in particular for those with greater filtering power, can cause the traumatism of an already pathological face region, with consequent worsening of the toxicity in progress. The continued use of gloves can worsen numerous dermatological toxicities affecting the hands of cancer patients (toxic erythema of chemotherapy, hand-foot syndrome, pruritus, acute paronychia, granuloma, etc.). Therefore, it is very important that cancer patients are followed up by their dermatologists even if the clinics are closed.

In order to guarantee a continuous support for oncological patients referred to our dedicated clinic for cutaneous adverse drug reactions, and to avoid possible exposure in high-risk settings such as hospitals, we are promoting the use of a crucial tool: teledermatology [9]. In fact, we have reserved access to the Dermatology Department only for urgent visits and, for all the others, we keep on using other means of communications such as emails and telephone, available $24 \mathrm{~h}$ a day, 7 days a week. The medical staff is also available for video calls to provide online dermatological consultations, and any requiring patient can easily and safely access this service. We also clarified the limits of the procedure to the patients and discriminated the cases that can be managed remotely and the cases that needed to be visited in person [10].

In order to preliminary assess the health status of oncological patients referred to our dedicated clinic, we contacted 105 patients either by telephone, video call, or email (Table 1). Among 87 participating patients, many had a stable or improving conditions $(n=53)$ and only few patients $(n=14)$ required either a shift or an increased dose of the therapy previously prescribed (Table 1). All the participating patients appreciated the survey very much.

As recommended for HCWs in China [8], we always stress the importance of skin and mucosa care (e.g., oral care) in addition to general prevention rules to all patients to limit as much as possible cutaneous or mucosal breakdowns that may aggravate AEs, predispose to secondary infections, and impair also patients' quality of life. 


\section{Compliance with ethical standards}

Conflict of interest The authors declare that they have no conflict of interest.

\section{References}

1. Livingston E, Bucher K (2020) Coronavirus disease 2019 (COVID19) in Italy. JAMA [Online ahead of print]

2. https://www.epicentro.iss.it/coronavirus/bollettino/Infografica 13aprile\%20ITA.pdf last accessed 14 April 2020

3. Marasca C, Ruggiero A, Annunziata MC, Fabbrocini G, Megna M (2020) Face the COVID-19 emergency: measures applied in an Italian dermatologic clinic. J Eur Acad Dermatol Venereol [In press]. https://doi.org/10.1111/jdv.16476

4. Liang W, Guan W, Chen R, Wang W, Li J, Xu K, Li C, Ai Q, Lu W, Liang H, Li S, He J (2020) Cancer patients in SARS-CoV-2 infection: a nationwide analysis in China. Lancet Oncol 21(3):335337
5. Xia Y, Jin R, Zhao J, Li W, Shen H (6) Risk of COVID-19 for cancer patients. Lancet Oncol [Online ahead of print]

6. Recalcati S (2020) Cutaneous manifestations in COVID-19: a first perspective. J Eur Acad Dermatol Venereol [Online ahead of print]

7. Annunziata MC, De Stefano A, Fabbrocini G et al (2019) Current recommendations and novel strategies for the management of skin toxicities related to anti-EGFR therapies in patients with metastatic colorectal cancer. Clin Drug Investig 39(9):825-834. https://doi. org/10.1007/s40261-019-00811-7

8. Yan Y, Chen H, Chen L, et al. (2020) Consensus of Chinese experts on protection of skin and mucous membrane barrier for healthcare workers fighting against coronavirus disease 2019. Dermatol Ther, e13310 [Online ahead of print]

9. Villani A, Scalvenzi M, Fabbrocini G (2020) Teledermatology: a useful tool to fight COVID-19. J Dermatolog Treat. 1. doi: https:// doi.org/10.1080/09546634.2020.1750557. Online ahead of print

10. Fattore D, Delfino M, Fabbrocini G (2019 Oct) Legal, medical, and ethical implications of instant messaging application use in dermatology. Int J Dermatol 58(10):e206-e207

Publisher's note Springer Nature remains neutral with regard to jurisdictional claims in published maps and institutional affiliations. 